\title{
TEICHMÜLLER SPACES OF GENERALIZED SYMMETRIC HOMEOMORPHISMS
}

\author{
HUAYING WEI AND KATSUHIKO MATSUZAKI \\ (Communicated by Jeremy Tyson)
}

\begin{abstract}
We introduce the concept of a new kind of symmetric homeomorphism on the unit circle, which is derived from the generalization of symmetric homeomorphisms on the real line. By the investigation of the barycentric extension for this class of circle homeomorphisms and the biholomorphic automorphisms induced by trivial Beltrami coefficients, we show that the Bers Schwarzian derivative map is a holomorphic split submersion and endow a complex Banach manifold structure on the Teichmüller space of those generalized symmetric homeomorphisms.
\end{abstract}

\section{INTRODUCTION}

The universal Teichmüller space plays a fundamental role in the quasiconformal theory of Teichmüller spaces, and it is also an important object in mathematical physics. The universal Teichmüller space $T$ can be defined as the group QS of all quasisymmetric homeomorphisms of the unit circle $\mathbb{S}=\{z \in \mathbb{C}|| z \mid=1\}$ modulo the left action of the group Möb $(\mathbb{S})$ of all Möbius transformations of $\mathbb{S}$, i.e., $T=\operatorname{Möb}(\mathbb{S}) \backslash$ QS. It can also be defined on the real line $\mathbb{R}$ by the conjugation of a Möbius transformation.

Several subclasses of quasisymmetric homeomorphisms and their Teichmüller spaces, spread in different directions, were introduced and studied for various purposes in the literature. We refer to the books [2, 11, 15, 17, and the papers [3, 6, 12, 16, 18, 20 for introducing these subject matters in more detail. Our work in this paper is mainly based on the subclass consisting of all symmetric homeomorphisms of $\mathbb{R}$ onto itself and is motivated by recent work of $\mathrm{Hu}, \mathrm{Wu}$, and Shen 13 .

A symmetric homeomorphism on the real line $\mathbb{R}$ was first studied in [5] when Carleson discussed absolute continuity of quasisymmetric homeomorphisms. It was proved that $h$ is symmetric if and only if $h$ can be extended to an asymptotically conformal homeomorphism $f$ of the upper half-plane $\mathbb{U}$ onto itself. Later, Gardiner and Sullivan 12 introduced the concept of the symmetric structure on $\mathbb{S}$ by relying on this relationship. By an asymptotically conformal homeomorphism $f$ of $\mathbb{U}$, we

Received by the editors May 29, 2019, and, in revised form, February 13, 2020.

2010 Mathematics Subject Classification. Primary 30F60, 30C62, 32G15; Secondary 37E10, $58 \mathrm{D} 05$.

Key words and phrases. Symmetric homeomorphism, asymptotic Teichmüller space, Bers embedding, barycentric extension.

This research was supported by the National Natural Science Foundation of China (Grant No. 11501259) and Japan Society for the Promotion of Science (KAKENHI 18H01125).

(C) 2020 by the authors under Creative Commons Attribution-Noncommercial 3.0 License (CC BY NC 3.0) 
mean that its complex dilatation $\mu=\bar{\partial} f / \partial f$ satisfies that

$$
\underset{y \leqslant t}{\operatorname{ess} \sup }|\mu(x+i y)| \rightarrow 0 \quad(t \rightarrow 0) .
$$

In fact, the Beurling-Ahlfors extension of $h$ is asymptotically conformal when $h$ is symmetric (see [5, 12, 16]). Based on these results, $\mathrm{Hu}, \mathrm{Wu}$, and Shen 13 endowed the symmetric Teichmüller space $T_{*}(\mathbb{R})$ on the real line with a complex Banach manifold structure. Namely, $T_{*}(\mathbb{R})$ can be embedded as a bounded domain in a certain Banach space.

The conjugation by the Cayley transformation $\phi_{\xi}(z)=\xi(z-i) /(z+i)$ that maps $\mathbb{R}$ onto $\mathbb{S}$ with $\infty \mapsto \xi$ transfers a symmetric self-homeomorphism $h$ of $\mathbb{R}$ to $\hat{h}=\phi_{\xi} \circ h \circ\left(\phi_{\xi}\right)^{-1}$ of $\mathbb{S}$. Then, we see that $\hat{h}$ extends to a quasiconformal self-homeomorphism of the unit disk $\mathbb{D}$ whose complex dilatation can be arbitrarily small outside some horoball tangent at $\xi$ in $\mathbb{D}$. In this paper, we generalize this transformation for only one tangent point to the case of plural tangent points by specifying a set $X$ of points $\xi \in \mathbb{S}$. We define the set $\mathrm{QS}_{*}^{X}$ of the generalized symmetric homeomorphisms for $X \subset \mathbb{S}$ by the boundary extension of quasiconformal self-homeomorphisms of $\mathbb{D}$ whose complex dilatations are arbitrarily small outside some horoballs tangent at all $\xi \in X$. We denote the set of such complex dilatations by $M_{*}^{X}(\mathbb{D})$. Then, we introduce the generalized symmetric Teichmüller space $T_{*}^{X}$ by $\mathrm{QS}_{*}^{X}$ modulo Möb(S) as well as the Teichmüller projection $\pi: M_{*}^{X}(\mathbb{D}) \rightarrow T_{*}^{X}$.

The Teichmüller space $T_{*}^{X}$ is of interest because it lies between the universal Teichmüller space $T$ and its little subspace $T_{0}=\operatorname{Möb}(\mathbb{S}) \backslash$ Sym made of the symmetric homeomorphisms of $\mathbb{S}$. We expect that a family $T_{*}^{X_{n}}$ defined by a sequence of increasing subsets $X_{n} \subset \mathbb{S}$ can give an interpolation between $T_{0}$ and $T$.

To investigate the structure of $T_{*}^{X}$, we show that the barycentric extension by Douady and Earle 7] gives a proper quasiconformal extension for the elements of $\mathrm{QS}_{*}^{X}$ to those whose complex dilatations are in $M_{*}^{X}(\mathbb{D})$. The proof is given by an adaptation of the argument by Earle, Markovic, and Saric 9]. After this, we can endow $T_{*}^{X}$ with a complex Banach manifold structure as a bounded domain in the corresponding Banach space $B_{*}^{X}\left(\mathbb{D}^{*}\right)$. The proof is carried out by using the Bers Schwarzian derivative map $\Phi: M_{*}^{X}(\mathbb{D}) \rightarrow B_{*}^{X}\left(\mathbb{D}^{*}\right)$ and showing that it is a holomorphic split submersion as usual. At this stage, the barycentric extension is also useful. Moreover, we point out that due to lack of a group structure on $M_{*}^{X}(\mathbb{D})$, we need some careful arguments for holomorphic split submersions different from the usual case.

\section{Preliminaries}

In this section, we review basic facts on the universal Teichmüller space and the symmetric Teichmüller space on the real line.

2.1. Universal Teichmüller space. We begin with a standard theory of the universal Teichmüller space. For details, we can refer to monographs [11,15, 17]. The universal Teichmüller space $T$ is a universal parameter space for the complex structures on all Riemann surfaces and can be defined as the space of all normalized quasisymmetric homeomorphisms on $\mathbb{S}$, namely, $T=\operatorname{Möb}(\mathbb{S}) \backslash$ QS. A topology of $T$ can be defined by quasisymmetry constants of quasisymmetric homeomorphisms. There are several ways to introduce quasisymmetric homeomorphisms on $\mathbb{S}$. In this paper, we lift $h: \mathbb{S} \rightarrow \mathbb{S}$ to $\tilde{h}: \mathbb{R} \rightarrow \mathbb{R}$ against the universal covering projection 
$\mathbb{R} \rightarrow \mathbb{S}$ with $x \mapsto e^{i x}$ and apply the definition of the quasisymmetry on $\mathbb{R}$ given in the next subsection.

The universal Teichmüller space $T$ can also be defined by using quasiconformal homeomorphisms $f$ of the unit disk $\mathbb{D}=\{z \in \mathbb{C}|| z \mid<1\}$ with complex dilatations $\mu_{f}=\bar{\partial} f / \partial f$ in the open unit ball $M(\mathbb{D})$ of the Banach space $L^{\infty}(\mathbb{D})$ of essentially bounded measurable functions on $\mathbb{D}$. More precisely, for $\mu \in M(\mathbb{D})$, the solution of the Beltrami equation (the measurable Riemann mapping theorem (see 2])) gives the unique quasiconformal homeomorphism $f^{\mu}$ of $\mathbb{D}$ onto itself that has complex dilatation $\mu$ and satisfies a certain normalization condition. This condition can be given by fixing three distinct points on $\mathbb{S}$ : for example, $1, i,-1$. We note that $f^{\mu}$ extends continuously to $\mathbb{S}$; the fixed point condition above is applied to this extension. This normalization cancels the freedom of post-composition of Möbius transformations. By giving the normalization, $M(\mathbb{D})$ becomes a group with operation $*$, where $\mu * \nu$ for $\mu, \nu \in M(\mathbb{D})$ is defined as the complex dilatation of $f^{\mu} \circ f^{\nu}$. The inverse $\nu^{-1}$ denotes the complex dilatation of $\left(f^{\nu}\right)^{-1}$.

It is known that the continuous extension of $f^{\mu}$ to $\mathbb{S}$, denoted by the same $f^{\mu}$, is a quasisymmetric homeomorphism of $\mathbb{S}$. Conversely, any normalized (i.e., keeping the points $1, i,-1$ fixed) quasisymmetric homeomorphism of $\mathbb{S}$ extends continuously to a quasiconformal homeomorphism $f^{\mu}$ of $\mathbb{D}$ for some $\mu \in M(\mathbb{D})$. We say that $\mu$ and $\nu$ in $M(\mathbb{D})$ are equivalent $(\mu \sim \nu)$ if $f^{\mu}=f^{\nu}$ on $\mathbb{S}$. We denote the equivalence class of $\mu$ by $[\mu]$. Then, the correspondence $\left.[\mu] \mapsto f^{\mu}\right|_{\mathbb{S}}$ establishes a bijection from $M(\mathbb{D}) / \sim$ onto $T$. Thus, the universal Teichmüller space $T$ is identified with $M(\mathbb{D}) / \sim$. The topology of $T=\operatorname{Möb}(\mathbb{S}) \backslash \mathrm{QS}$ coincides with the quotient topology induced by the Teichmüller projection $\pi: M(\mathbb{D}) \rightarrow T$.

The universal Teichmüller space $T$ is also identified with a domain in the Banach space

$$
B\left(\mathbb{D}^{*}\right)=\left\{\varphi\left|\|\varphi\|_{B}=\sup _{z \in \mathbb{D}^{*}} \rho_{\mathbb{D}^{*}}^{-2}(z)\right| \varphi(z) \mid<\infty\right\}
$$

of bounded holomorphic quadratic differentials $\varphi=\varphi(z) d z^{2}$ on $\mathbb{D}^{*}=\widehat{\mathbb{C}}-\overline{\mathbb{D}}$ under the Bers embedding $\beta: T \rightarrow B\left(\mathbb{D}^{*}\right)$. Here, $\rho_{\mathbb{D}^{*}}(z)=\left(|z|^{2}-1\right)^{-1}$ denotes the hyperbolic density on $\mathbb{D}^{*}$. This map is given by the factorization of a map $\Phi$ : $M(\mathbb{D}) \rightarrow B\left(\mathbb{D}^{*}\right)$ by the Teichmüller projection $\pi$, i.e., $\beta \circ \pi=\Phi$. Here, for every $\mu \in M(\mathbb{D}), \Phi(\mu)$ is defined by the Schwarzian derivative $\mathcal{S}\left(\left.f_{\mu}\right|_{\mathbb{D}^{*}}\right)$, where $f_{\mu}$ is a quasiconformal homeomorphism of the complex plane $\widehat{\mathbb{C}}$ that has complex dilatation $\mu$ in $\mathbb{D}$ and is conformal in $\mathbb{D}^{*}$. The map $\Phi$ is called the Bers Schwarzian derivative map.

The Bers embedding $\beta: T \rightarrow B\left(\mathbb{D}^{*}\right)$ is a homeomorphism onto the image $\beta(T)=$ $\Phi(M(\mathbb{D}))$, and it defines a complex structure of $T$ as a domain in the Banach space $B\left(\mathbb{D}^{*}\right)$. It is proved that $\Phi$ (and so also $\pi$ ) is a holomorphic split submersion from $M(\mathbb{D})$ onto its image.

The barycentric extension in Douady and Earle [7] gives a quasiconformal extension $E(h) \in \mathrm{QC}(\mathbb{D})$ of a quasisymmetric homeomorphism $h \in \mathrm{QS}$ in a conformally natural way. This means that $E\left(g_{1} \circ h \circ g_{2}\right)=E\left(g_{1}\right) \circ E(h) \circ E\left(g_{2}\right)$ is satisfied for any $h \in \mathrm{QS}$ and any $g_{1}, g_{2} \in \operatorname{Möb}(\mathbb{S})$, where the extensions $E\left(g_{1}\right)$ and $E\left(g_{2}\right)$ are conformal (Möbius) on $\mathbb{D}$. The quasiconformal extension $E(h)$ is a diffeomorphism of $\mathbb{D}$ that is bi-Lipschitz with respect to the hyperbolic metric. The barycentric extension induces a continuous (in fact, real analytic) section $s: T \rightarrow M(\mathbb{D})$ of the Teichmüller projection $\pi: M(\mathbb{D}) \rightarrow T\left(\pi \circ s=\mathrm{id}_{T}\right)$ by sending a point $[\mu] \in T$ to the complex dilatation $s([\mu]) \in M(\mathbb{D})$ of $E\left(\left.f^{\mu}\right|_{\mathbb{S}}\right)$. By the conformal naturality of 
the barycentric extension, the Teichmüller space of any Fuchsian group is shown to be contractible.

2.2. Symmetric Teichmüller space on the real line. An increasing homeomorphism $h$ of the real line $\mathbb{R}$ onto itself is said to be quasisymmetric if there exists some $M \geqslant 1$ such that

$$
\frac{1}{M} \leqslant m_{h}(x, t):=\frac{h(x+t)-h(x)}{h(x)-h(x-t)} \leqslant M
$$

for all $x \in \mathbb{R}$ and $t>0$. The optimal value of such $M$ is called the quasisymmetry constant for $h$.

Beurling and Ahlfors [4 proved that $h$ is quasisymmetric if and only if there exists some quasiconformal homeomorphism of the upper half-plane $\mathbb{U}=\{x+i y \in$ $\mathbb{C} \mid y>0\}$ onto itself that is continuously extendable to the boundary map $h$. Let $\mathrm{QS}(\mathbb{R})$ denote the group of all quasisymmetric homeomorphisms of $\mathbb{R}$.

A quasisymmetric homeomorphism $h$ is said to be symmetric if $m_{h}(x, t) \rightarrow 1$ uniformly as $t \rightarrow 0$ for all $x \in \mathbb{R}$. Let $\mathrm{QS}_{*}(\mathbb{R})$ denote the subset of $\mathrm{QS}(\mathbb{R})$ (in fact, this is not a subgroup as shown in [21]) consisting of all symmetric homeomorphisms of $\mathbb{R}$. It is known that $h$ is symmetric if and only if $h$ can be extended to an asymptotically conformal homeomorphism $f$ of $\mathbb{U}$ onto itself (see [12]). In fact, the Beurling-Ahlfors extension of $h$ is asymptotically conformal when $h$ is symmetric. By an asymptotically conformal homeomorphism $f$ of $\mathbb{U}$, we mean that its complex dilatation $\nu_{f}=\bar{\partial} f / \partial f$ belongs to $M_{*}(\mathbb{U})=L_{*}(\mathbb{U}) \cap M(\mathbb{U})$, where $M(\mathbb{U})$ is the open unit ball of $L^{\infty}(\mathbb{U})$ and

$$
L_{*}(\mathbb{U})=\left\{\nu \in L^{\infty}(\mathbb{U}) \mid \forall \epsilon>0, \exists t>0 \quad \text { such that }\left\|\left.\nu\right|_{\mathbb{U} \backslash H_{t}}\right\|_{\infty}<\epsilon\right\} .
$$

Here, $H_{t}=\{x+i y \in \mathbb{U} \mid y \geqslant t\}(t>0)$ is a horoplane tangent at $\infty$.

We define $T_{*}(\mathbb{R})=\operatorname{Aff}(\mathbb{R}) \backslash \mathrm{QS}_{*}(\mathbb{R})$ as the symmetric Teichmüller space on the real line $\mathbb{R}$, where $\operatorname{Aff}(\mathbb{R})$ denotes the subgroup of all real affine mappings $z \mapsto a z+b$, $a>0, b \in \mathbb{R}$. Recently, $\mathrm{Hu}, \mathrm{Wu}$, and Shen [13] endowed $T_{*}(\mathbb{R})$ with a complex Banach manifold structure modeled on the closed subspace $B_{*}(\mathbb{L})$ of the Banach space

$$
B(\mathbb{L})=\left\{\psi\left|\|\psi\|_{B}=\sup _{z \in \mathbb{L}} \rho_{\mathbb{L}}^{-2}(z)\right| \psi(z) \mid<\infty\right\}
$$

of bounded holomorphic quadratic differentials on the lower half-plane $\mathbb{L}=\{x+i y \in$ $\mathbb{C} \mid y<0\}$, which consists of those $\psi$ satisfying that for any $\varepsilon>0$, there exists $t>0$ such that

$$
\sup _{z \in \mathbb{L} \backslash H_{t}^{*}}|\psi(z)| \rho_{\mathbb{L}}^{-2}(z)<\varepsilon .
$$

Here, $\rho_{\mathbb{L}}(z)=(2 \operatorname{Im} z)^{-1}$ is the hyperbolic density on $\mathbb{L}$, and $H_{t}^{*}=\{x+i y \in \mathbb{L} \mid$ $-y \geqslant t\}$ is the reflection of the horoplane $H_{t}$ with respect to $\mathbb{R}$.

A quasisymmetric homeomorphism $h \in \mathrm{QS}$ of $\mathbb{S}$ is also called symmetric if its lift $\tilde{h}: \mathbb{R} \rightarrow \mathbb{R}$ is symmetric in the above sense. We denote the subgroup of QS consisting of all symmetric homeomorphisms of $\mathbb{S}$ by Sym. Then, the little universal Teichmüller space is defined by $T_{0}=\operatorname{Möb}(\mathbb{S}) \backslash \operatorname{Sym}$ and has been studied in the theory of asymptotic Teichmüller spaces (see [8,12]). The universal Teichmüller space can also be defined on the real line by $T(\mathbb{R})=\operatorname{Aff}(\mathbb{R}) \backslash \mathrm{QS}(\mathbb{R})$, and this is isomorphic to $T=\operatorname{Möb}(\mathbb{S}) \backslash \mathrm{QS}$ under the conjugation by the Cayley transformation. We note however that $T_{*}(\mathbb{R})$ is not isomorphic to $T_{0}$ under this isomorphism $T(\mathbb{R}) \cong$ $T$ (see [13]). 


\section{Generalized symmetric Teichmüller space}

In this section, we will introduce generalized symmetric homeomorphisms and the generalized symmetric Teichmüller space by transferring $\mathrm{QS}_{*}(\mathbb{R})$ to the unit circle $\mathbb{S}$ and extending it to the general case by specifying a set of points $\xi \in \mathbb{S}$. We also transfer $M_{*}(\mathbb{U})$ to the unit disk $\mathbb{D}$ and $B_{*}(\mathbb{L})$ to the exterior of the unit disk $\mathbb{D}^{*}$.

For every $\xi \in \mathbb{S}$, let $\phi_{\xi}(z)=\xi(z-i) /(z+i)$ be the Cayley transformation of $\mathbb{U}$ onto $\mathbb{D}$ that sends $\infty$ to $\xi$ and $i$ to 0 . Then, the push-forward operator $\left(\phi_{\xi}\right)_{*}: L^{\infty}(\mathbb{U}) \rightarrow L^{\infty}(\mathbb{D})$ defined by

$$
\left(\phi_{\xi}\right)_{*} \nu=\nu \circ \phi_{\xi}^{-1} \overline{\left(\phi_{\xi}^{-1}\right)^{\prime}} /\left(\phi_{\xi}^{-1}\right)^{\prime}
$$

for every $\nu \in L^{\infty}(\mathbb{U})$ is a linear isometry. Let $L_{*}^{\xi}(\mathbb{D})=\left(\phi_{\xi}\right)_{*}\left(L_{*}(\mathbb{U})\right)$ and $M_{*}^{\xi}(\mathbb{D})=$ $\left(\phi_{\xi}\right)_{*}\left(M_{*}(\mathbb{U})\right)$. Clearly, $M_{*}^{\xi}(\mathbb{D})=L_{*}^{\xi}(\mathbb{D}) \cap M(\mathbb{D})$.

For our purpose of generalization, we represent $L_{*}^{\xi}(\mathbb{D})$ as follows. We consider a horoball $D_{t}^{\xi}=\phi_{\xi}\left(H_{t}\right)$ for $t>0$, which is tangent at $\xi$ in $\mathbb{D}$ with the boundary

$$
\partial D_{t}^{\xi}=\left\{\xi \frac{x+i(t-1)}{x+i(t+1)} \in \mathbb{D} \mid x \in \mathbb{R}\right\} .
$$

Then, it is clear that

$$
L_{*}^{\xi}(\mathbb{D})=\left\{\mu \in L^{\infty}(\mathbb{D}) \mid \forall \epsilon>0, \exists t>0 \quad \text { such that }\left\|\left.\mu\right|_{\mathbb{D} \backslash D_{t}^{\xi}}\right\|_{\infty}<\epsilon\right\} .
$$

Now we extend the definition above for only one tangent point to the case of plural tangent points. Let $X=\left\{\xi_{1}, \xi_{2}, \ldots, \xi_{n}\right\}$ be a finite subset of $\mathbb{S}$. Let

$$
L_{*}^{X}(\mathbb{D})=\left\{\mu \in L^{\infty}(\mathbb{D}) \mid \forall \epsilon>0, \exists t>0 \quad \text { such that }\left\|\left.\mu\right|_{\mathbb{D} \backslash \bigcup_{i=1}^{n} D_{t}^{\xi_{i}}}\right\|_{\infty}<\epsilon\right\}
$$

and $M_{*}^{X}(\mathbb{D})=L_{*}^{X}(\mathbb{D}) \cap M(\mathbb{D})$. We see that $L_{*}^{X}(\mathbb{D})$ is closed in $L^{\infty}(\mathbb{D})$. Indeed, assuming that a sequence $\left\{\mu_{k}\right\}_{k \in \mathbb{N}}$ in $L_{*}^{X}(\mathbb{D})$ and $\mu \in L^{\infty}(\mathbb{D})$ are given so that $\left\|\mu_{k}-\mu\right\|_{\infty} \rightarrow 0$ as $k \rightarrow \infty$, we show that $\mu \in L_{*}^{X}(\mathbb{D})$. For each $\varepsilon>0$, we can choose some $k_{0} \in \mathbb{N}$ such that $\left\|\mu_{k_{0}}-\mu\right\|_{\infty}<\varepsilon$. Since $\mu_{k_{0}} \in L_{*}^{X}(\mathbb{D})$, there exists some $t>0$ such that $\left\|\left.\mu_{k_{0}}\right|_{\mathbb{D} \backslash \bigcup_{i=1}^{n} D_{t}^{\xi_{i}}}\right\|_{\infty}<\varepsilon$. Thus,

$$
\left\|\left.\mu\right|_{\mathbb{D} \backslash \bigcup_{i=1}^{n} D_{t}^{\xi_{i}}}\right\|_{\infty} \leqslant\left\|\left.\mu_{k_{0}}\right|_{\mathbb{D} \backslash \bigcup_{i=1}^{n} D_{t}^{\xi_{i}}}\right\|_{\infty}+\left\|\mu_{k_{0}}-\mu\right\|_{\infty}<2 \varepsilon,
$$

which implies that $\mu \in L_{*}^{X}(\mathbb{D})$.

Here, we note the following fact on an algebraic structure of the space of the Beltrami differentials.

Proposition 3.1. For any $X=\left\{\xi_{1}, \ldots, \xi_{n}\right\} \subset \mathbb{S}, L_{*}^{X}(\mathbb{D})=L_{*}^{\xi_{1}}(\mathbb{D})+\cdots+L_{*}^{\xi_{n}}(\mathbb{D})$.

Proof. The inclusion $\supset$ is easy to see. For the inverse inclusion $\subset$, we take any element $\mu$ in $L_{*}^{X}(\mathbb{D})$. The unit circle $\mathbb{S}$ is divided into $n$ subarcs by the points $\xi_{1}, \ldots, \xi_{n}$. We take the midpoint of each subarc and connect it to the origin 0 by a segment. The union of these segments divides $\mathbb{D}$ into $n$ sectors $E_{1}, \ldots, E_{n}$, and each $E_{i}(i=1, \ldots, n)$ contains only one $\xi_{i}$ on its boundary. Then, the decomposition of $\mu$ is given simply by restricting $\mu$ to each sector, $\mu=\mu 1_{E_{1}}+\cdots+\mu 1_{E_{n}}$, where $\mu 1_{E_{i}} \in L_{*}^{\xi_{i}}(\mathbb{D})$ for each $i=1, \ldots, n$.

This implies that

$$
M_{*}^{X}(\mathbb{D})=\left(L_{*}^{\xi_{1}}(\mathbb{D})+\cdots+L_{*}^{\xi_{n}}(\mathbb{D})\right) \cap M(\mathbb{D}) .
$$


For $\mu \in M_{*}^{X}(\mathbb{D})$, a quasisymmetric homeomorphism obtained by the boundary extension of a quasiconformal homeomorphism of $\mathbb{D}$ onto itself with dilatation $\mu$ is called a generalized symmetric homeomorphism for $X$. The subset of QS consisting of all such elements is denoted by $\mathrm{QS}_{*}^{X}$. We remark that this is not a subgroup of QS.

Definition 3.2. Let $X \subset \mathbb{S}$ be a finite subset. The generalized symmetric Teichmüller space $T_{*}^{X}$ for $X$ is defined as

$$
T_{*}^{X}=\operatorname{Möb}(\mathbb{S}) \backslash \mathrm{QS}_{*}^{X}=\pi\left(M_{*}^{X}(\mathbb{D})\right) .
$$

Remark 3.3. The Teichmüller spaces $T$ and $T_{0}$ have a group structure by the composition of the normalized elements of QS. However, $T_{*}^{X}$ is not a subgroup of $T$ even if $X$ consists of only one point. See [21].

We can also characterize a generalized symmetric homeomorphism $h$ for $X$ as the mapping on $\mathbb{S}$. For an asymptotically conformal homeomorphism $f$ of $\mathbb{U}$ and the corresponding symmetric homeomorphism $h$ of $\mathbb{R}$, if $\left\|\left.\mu_{f}\right|_{\mathbb{U} \backslash H_{t}}\right\|_{\infty} \rightarrow 0$ as $t \rightarrow 0$, then $\sup _{x \in \mathbb{R}, 0<s<t}\left|m_{h}(x, s)-1\right| \rightarrow 0$. If $f$ is given by the Beurling-Ahlfors extension of $h$, then the converse implication is also true. These results were proved by Carleson [5] (see also [16. Theorems 5.1 and 5.2]). Examining their proofs, we also see that this correspondence can be localized (see also [10, Section 3]). Then, we translate the situations into those on the unit disk $\mathbb{D}$ and the unit circle $\mathbb{S}$ by the Cayley transformation $\phi_{\xi}$ sending $\infty$ to any point $\xi$ in a given set $X \subset \mathbb{S}$. In this case, the interval $t$ on $\mathbb{R}$ should be scaled to $d(x)^{2} t$ at $e^{i x} \in \mathbb{S} \backslash X$, where $d(x)$ is the distance from $e^{i x}$ to $X$ on $\mathbb{S}$. We note that $d(x)^{2} t$ is comparable to the Euclidean distance from $e^{i x} \in \mathbb{S}$ to the union of the horoballs $\bigcup_{\xi \in X} D_{t}^{\xi}$. Therefore, for a quasisymmetric homeomorphism $h$ of $\mathbb{S}$, the condition $h \in \mathrm{QS}_{*}^{X}$ is equivalent to the following: for any $\varepsilon>0$, there is $\delta>0$ such that if $e^{i x} \in \mathbb{S} \backslash X$ and $s<d(x)^{2} \delta$, then

$$
\left|\frac{h\left(e^{i(x+s)}\right)-h\left(e^{i x}\right)}{h\left(e^{i x}\right)-h\left(e^{i(x-s)}\right)}-1\right|<\varepsilon .
$$

\section{Bers Schwarzian Derivative map}

In this section, we focus on the Bers Schwarzian derivative map $\Phi: M(\mathbb{D}) \rightarrow$ $B\left(\mathbb{D}^{*}\right)$ restricted to the subspace $M_{*}^{X}(\mathbb{D})$. We first introduce the corresponding subspace of $B\left(\mathbb{D}^{*}\right)$.

For this purpose, we use the same Cayley transformation $\phi_{\xi}(z)=\xi(z-i) /(z+i)$ as before for every $\xi \in \mathbb{S}$. This maps $\mathbb{L}$ onto $\mathbb{D}^{*}$ sending $\infty$ to $\xi$ and $-i$ to $\infty$. The push-forward operator $\left(\phi_{\xi}\right)_{*}: B(\mathbb{L}) \rightarrow B\left(\mathbb{D}^{*}\right)$ defined by

$$
\left(\phi_{\xi}\right)_{*} \psi=\psi \circ \phi_{\xi}^{-1}\left(\phi_{\xi}^{-1}\right)^{\prime 2}
$$

for every $\psi \in B(\mathbb{L})$ is a linear isometry. Let $B_{*}^{\xi}\left(\mathbb{D}^{*}\right)=\left(\phi_{\xi}\right)_{*}\left(B_{*}(\mathbb{L})\right)$.

We consider a horoball $\left(D_{t}^{\xi}\right)^{*}=\phi_{\xi}\left(H_{t}^{*}\right)$ tangent at $\xi$ in $\mathbb{D}^{*}$ such that

$$
\partial\left(D_{t}^{\xi}\right)^{*}=\left\{\xi \frac{x+i(t+1)}{x+i(t-1)} \in \mathbb{D}^{*} \mid x \in \mathbb{R}\right\} .
$$

This is the reflection of $D_{t}^{\xi}$ with respect to $\mathbb{S}$. Then, we see that

$$
B_{*}^{\xi}\left(\mathbb{D}^{*}\right)=\left\{\varphi \in B\left(\mathbb{D}^{*}\right) \mid \forall \varepsilon>0, \exists t>0 \quad \text { such that }\left\|\left.\varphi\right|_{\mathbb{D}^{*} \backslash\left(D_{t}^{\xi}\right)^{*}}\right\|_{B}<\varepsilon\right\} .
$$


For a finite subset $X=\left\{\xi_{1}, \xi_{2}, \ldots, \xi_{n}\right\} \subset \mathbb{S}$, we also generalize this to

$$
B_{*}^{X}\left(\mathbb{D}^{*}\right)=\left\{\varphi \in B\left(\mathbb{D}^{*}\right) \mid \forall \varepsilon>0, \exists t>0 \quad \text { such that }\left\|\left.\varphi\right|_{\mathbb{D}^{*} \backslash \bigcup_{i=1}^{n}\left(D_{t}^{\xi_{i}}\right)^{*}}\right\|_{B}<\varepsilon\right\},
$$

which is the desired Banach subspace of $B\left(\mathbb{D}^{*}\right)$.

By the following theorem, we see that $B_{*}^{X}\left(\mathbb{D}^{*}\right)$ is the appropriate space corresponding to $M_{*}^{X}(\mathbb{D})$ under the Bers Schwarzian derivative map $\Phi$.

Theorem 4.1. For every finite subset $X \subset \mathbb{S}$, the Bers Schwarzian derivative map $\Phi \operatorname{maps} M_{*}^{X}(\mathbb{D})$ into $B_{*}^{X}\left(\mathbb{D}^{*}\right)$.

Proof. By the integral representation of the Schwarzian derivative, which was established by Astala and Zinsmeister [3] (see also Cui [6]), we have that for $\zeta^{*} \in \mathbb{D}^{*}$,

$$
\rho_{\mathbb{D}^{*}}^{-4}\left(\zeta^{*}\right)\left|\Phi(\mu)\left(\zeta^{*}\right)\right|^{2} \leqslant C \int_{\mathbb{D}} \frac{\left(\left|\zeta^{*}\right|^{2}-1\right)^{2}}{\left|z-\zeta^{*}\right|^{4}}|\mu(z)|^{2} d x d y,
$$

where $C>0$ is a constant depending only on $\|\mu\|_{\infty}$.

Let $\gamma_{\zeta}(z)=\left(\overline{\zeta^{*}} z-1\right) /\left(z-\zeta^{*}\right) \in \operatorname{Möb}(\mathbb{D})$ be a Möbius transformation of $\mathbb{D}$ onto itself that sends $\zeta$ to 0 . Here, $\zeta \in \mathbb{D}$ and $\zeta^{*} \in \mathbb{D}^{*}$ are the reflection points to each other with respect to $\mathbb{S}$. We see that $\left|\gamma_{\zeta}^{\prime}(z)\right|^{2}=\left(\left|\zeta^{*}\right|^{2}-1\right)^{2} /\left|z-\zeta^{*}\right|^{4}$. It follows that

$$
\begin{aligned}
& \int_{\mathbb{D}} \frac{\left(\left|\zeta^{*}\right|^{2}-1\right)^{2}}{\left|z-\zeta^{*}\right|^{4}}|\mu(z)|^{2} d x d y=\int_{\mathbb{D}}\left|\gamma_{\zeta}^{\prime}(z)\right|^{2}|\mu(z)|^{2} d x d y \\
= & \int_{\mathbb{D} \backslash \bigcup_{i=1}^{n} D_{t}^{\xi_{i}}}\left|\gamma_{\zeta}^{\prime}(z)\right|^{2}|\mu(z)|^{2} d x d y+\sum_{i=1}^{n} \int_{D_{t}^{\xi_{i}}}\left|\gamma_{\zeta}^{\prime}(z)\right|^{2}|\mu(z)|^{2} d x d y .
\end{aligned}
$$

Here, for a given $\varepsilon>0$, we choose $t>0$ so that $\left\|\left.\mu\right|_{\mathbb{D} \backslash \bigcup_{i=1}^{n} D_{t}^{\xi_{i}}}\right\|_{\infty}<\varepsilon$ under the condition $\mu \in M_{*}^{X}(\mathbb{D})$. Then, the last formula is estimated from above by

$$
\begin{aligned}
& \varepsilon^{2} \int_{\mathbb{D} \backslash \bigcup_{i=1}^{n} D_{t}^{\xi_{i}}}\left|\gamma_{\zeta}^{\prime}(z)\right|^{2} d x d y+\sum_{i=1}^{n} \int_{D_{t}^{\xi_{i}}}\left|\gamma_{\zeta}^{\prime}(z)\right|^{2} d x d y \\
\leqslant & \pi \varepsilon^{2}+\sum_{i=1}^{n} \operatorname{Area}\left(\gamma_{\zeta}\left(D_{t}^{\xi_{i}}\right)\right),
\end{aligned}
$$

where Area stands for the Euclidean area.

We consider $\operatorname{Area}\left(\gamma_{\zeta}\left(D_{t}^{\xi_{i}}\right)\right)$. The notation $\asymp$ is used below when both sides are comparable; i.e., one side is bounded from above and below by multiples of the other side with some positive absolute constants. By $\operatorname{Area}\left(\gamma_{\zeta}\left(D_{t}^{\xi_{i}}\right)\right) \asymp \operatorname{diam}^{2}\left(\gamma_{\zeta}\left(D_{t}^{\xi_{i}}\right)\right)$ and $d_{H}(0, z)=\log \frac{1+|z|}{1-|z|}(z \in \mathbb{D})$ for the Euclidean diameter diam and the hyperbolic distance $d_{H}$, we see that

$$
\operatorname{diam}^{2}\left(\gamma_{\zeta}\left(D_{t}^{\xi_{i}}\right)\right) \asymp e^{-2 d_{H}\left(0, \gamma_{\zeta}\left(D_{t}^{\xi_{i}}\right)\right)}=e^{-2 d_{H}\left(\zeta, D_{t}^{\xi_{i}}\right)},
$$

and hence, the condition $\operatorname{Area}\left(\gamma_{\zeta}\left(D_{t}^{\xi_{i}}\right)\right) \leqslant \varepsilon^{2}$ is equivalent to that $d_{H}\left(\zeta, D_{t}^{\xi_{i}}\right) \geqslant$ $-\log \varepsilon$ up to some additive constant. We note that $d_{H}\left(\zeta, D_{t}^{\xi_{i}}\right)=d_{H}\left(\zeta^{*},\left(D_{t}^{\xi_{i}}\right)^{*}\right)$ by reflection and that the hyperbolic $a$-neighborhood $N_{a}\left(D_{t}^{\xi_{i}}\right)$ of $D_{t}^{\xi_{i}}$ is

$$
N_{a}\left(D_{t}^{\xi_{i}}\right)=\phi_{\xi_{i}}\left(N_{a}\left(H_{t}\right)\right)=\phi_{\xi_{i}}\left(H_{e^{-a} t}\right)=D_{e^{-a} t}^{\xi_{i}} .
$$

Thus, the condition $d_{H}\left(\zeta, D_{t}^{\xi_{i}}\right) \geqslant-\log \varepsilon$ is equivalent to that $\zeta^{*} \notin\left(D_{\varepsilon t}^{\xi_{i}}\right)^{*}$. This implies that if $\zeta^{*} \in \mathbb{D}^{*} \backslash \bigcup_{i=1}^{n}\left(D_{\varepsilon t}^{\xi_{i}}\right)^{*}$, then $\operatorname{Area}\left(\gamma_{\zeta}\left(D_{t}^{\xi_{i}}\right)\right) \leqslant A \varepsilon^{2}$ for some absolute constant $A$. 
We plug this area estimate into the above inequality. The conclusion is that if $\zeta^{*} \in \mathbb{D}^{*} \backslash \bigcup_{i=1}^{n}\left(D_{\varepsilon t}^{\xi_{i}}\right)^{*}$, then

$$
\rho_{\mathbb{D}^{*}}^{-2}\left(\zeta^{*}\right)\left|\Phi(\mu)\left(\zeta^{*}\right)\right| \leqslant \sqrt{C(\pi+A n)} \varepsilon .
$$

Since $\varepsilon>0$ is arbitrarily chosen, this implies that if $\mu \in M_{*}^{X}(\mathbb{D})$, then $\Phi(\mu) \in$ $B_{*}^{X}\left(\mathbb{D}^{*}\right)$.

\section{BARYCENTRIC EXTENSION}

In this section, we will prove that the barycentric extension introduced by Douady and Earle [7] gives an appropriate right inverse of $\pi: M_{*}^{X}(\mathbb{D}) \rightarrow T_{*}^{X}$ from the generalized symmetric Teichmüller space $T_{*}^{X}$ to the space $M_{*}^{X}(\mathbb{D})$ of the complex dilatations. In other words, for the section $s: T \rightarrow M(\mathbb{D})$ of the universal Teichmüller space induced by the barycentric extension, we show that the image $s\left(T_{*}^{X}\right)$ is in $M_{*}^{X}(\mathbb{D})$.

This claim follows from the following more general result concerning the section $s$. This was originally proved by Earle, Markovic, and Saric [9, Theorem 4] for the little universal Teichmüller space $T_{0}=\operatorname{Möb}(\mathbb{S}) \backslash$ Sym and for the subspaces $M_{0}(\mathbb{D}) \subset M(\mathbb{D})$ and $B_{0}\left(\mathbb{D}^{*}\right) \subset B\left(\mathbb{D}^{*}\right)$ consisting of the vanishing elements at the boundary. The proof below is a modification of theirs. In the proof, the pull-back of $\mu \in M(\mathbb{D})$ by $g \in \operatorname{Möb}(\mathbb{D})$ is defined by $g^{*} \mu(z)=\mu(g(z)) \overline{g^{\prime}(z)} / g^{\prime}(z)$, and the pull-back of $\varphi \in B\left(\mathbb{D}^{*}\right)$ by $g \in \operatorname{Möb}\left(\mathbb{D}^{*}\right)$ is defined by $g^{*} \varphi(z)=\varphi(g(z)) g^{\prime}(z)^{2}$.

Theorem 5.1. Let $\mu$ and $\nu$ be in $M(\mathbb{D})$. Let $X$ be a finite subset of $\mathbb{S}$. Then, the following are equivalent:

(1) $s([\mu])-s([\nu]) \in L_{*}^{X}(\mathbb{D})$;

(2) $\Phi(\mu)-\Phi(\nu) \in B_{*}^{X}\left(\mathbb{D}^{*}\right)$.

Proof. $(2) \Rightarrow(1)$ : We take an arbitrary sequence $\left\{z_{k}\right\}_{k \in \mathbb{N}} \subset \mathbb{D}$ such that $z_{k} \in$ $\mathbb{D} \backslash \bigcup_{i=1}^{n} D_{1 / k}^{\xi_{i}}$ for every $k \in \mathbb{N}$. For each $k$, we choose a Möbius transformation $g_{k} \in \operatorname{Möb}(\mathbb{D})$ with $g_{k}(0)=z_{k}$ and define $\mu_{k}=g_{k}^{*} s([\mu])$ and $\nu_{k}=g_{k}^{*} s([\nu])$. Then, $\Phi\left(\mu_{k}\right)=g_{k}^{*} \Phi(\mu)$ and $\Phi\left(\nu_{k}\right)=g_{k}^{*} \Phi(\nu)$ for $g_{k} \in \operatorname{Möb}\left(\mathbb{D}^{*}\right)$. We also see that for every $\tilde{k} \in \mathbb{N}$ and for every $z^{*} \in \mathbb{D}^{*}$, there is some $k_{0}$ such that $g_{k}\left(z^{*}\right) \in \mathbb{D}^{*} \backslash \bigcup_{i=1}^{n}\left(D_{1 / \tilde{k}}^{\xi_{i}}\right)^{*}$ for all $k \geqslant k_{0}$. Since we assume that $\Phi(\mu)-\Phi(\nu) \in B_{*}^{X}\left(\mathbb{D}^{*}\right)$, we have that

$$
\rho_{\mathbb{D}^{*}}^{-2}\left(z^{*}\right)\left|\Phi\left(\mu_{k}\right)\left(z^{*}\right)-\Phi\left(\nu_{k}\right)\left(z^{*}\right)\right|=\rho_{\mathbb{D}^{*}}^{-2}\left(g_{k}\left(z^{*}\right)\right)\left|(\Phi(\mu)-\Phi(\nu))\left(g_{k}\left(z^{*}\right)\right)\right|
$$

tends to 0 as $k \rightarrow \infty$ for each $z^{*} \in \mathbb{D}^{*}$. In particular, $\Phi\left(\mu_{k}\right)-\Phi\left(\nu_{k}\right) \rightarrow 0$ as $k \rightarrow \infty$.

Since $\left\|\mu_{k}\right\|_{\infty}=\|s([\mu])\|_{\infty}$ and $\left\|\nu_{k}\right\|_{\infty}=\|s([\nu])\|_{\infty}$, by passing to a subsequence, we may assume that $f^{\mu_{k}}$ converges uniformly to some quasiconformal homeomorphism $f^{\mu_{0}} \in \mathrm{QC}(\mathbb{D})$ with a complex dilatation $\mu_{0} \in M(\mathbb{D})$ and $f^{\nu_{k}}$ converges uniformly to some $f^{\nu_{0}} \in \mathrm{QC}(\mathbb{D})$ with $\nu_{0} \in M(\mathbb{D})$. In this situation, 9, Lemma 6.1] asserts that $\Phi\left(\mu_{k}\right)$ converges locally uniformly to $\Phi\left(\mu_{0}\right)$ and $\Phi\left(\nu_{k}\right)$ converges locally uniformly to $\Phi\left(\nu_{0}\right)$ on $\mathbb{D}^{*}$. Since $\Phi\left(\mu_{k}\right)-\Phi\left(\nu_{k}\right) \rightarrow 0$ as $k \rightarrow \infty$, this implies that $\Phi\left(\mu_{0}\right)=\Phi\left(\nu_{0}\right)$.

By [9, Lemma 6.1] again, we see that $s\left(\left[\mu_{k}\right]\right)$ converges locally uniformly to $s\left(\left[\mu_{0}\right]\right)$ and $s\left(\left[\nu_{k}\right]\right)$ converges locally uniformly to $s\left(\left[\nu_{0}\right]\right)$ on $\mathbb{D}$. Here, $\Phi\left(\mu_{0}\right)=\Phi\left(\nu_{0}\right)$ implies that $s\left(\left[\mu_{0}\right]\right)=s\left(\left[\nu_{0}\right]\right)$. Therefore, $s\left(\left[\mu_{k}\right]\right)-s\left(\left[\nu_{k}\right]\right)$ converges to 0 , and in particular, $s\left(\left[\mu_{k}\right]\right)(0)-s\left(\left[\nu_{k}\right]\right)(0) \rightarrow 0$ as $k \rightarrow \infty$.

The conformal naturality of the barycentric extension implies that

$$
s\left(\left[\mu_{k}\right]\right)=s\left(\left[g_{k}^{*} \mu\right]\right)=g_{k}^{*}(s([\mu])) ; \quad s\left(\left[\nu_{k}\right]\right)=s\left(\left[g_{k}^{*} \nu\right]\right)=g_{k}^{*}(s([\nu])) .
$$


It follows that

$$
\left|s([\mu])\left(z_{k}\right)-s([\nu])\left(z_{k}\right)\right|=\left|s\left(\left[\mu_{k}\right]\right)(0)-s\left(\left[\nu_{k}\right]\right)(0)\right| \rightarrow 0 \quad(k \rightarrow \infty) .
$$

Since $z_{k} \in \mathbb{D} \backslash \bigcup_{i=1}^{n} D_{1 / k}^{\xi_{i}}$ for every $k \in \mathbb{N}$, we see that $s([\mu])-s([\nu]) \in L_{*}^{X}(\mathbb{D})$.

$(1) \Rightarrow(2)$ : We take an arbitrary sequence $\left\{z_{k}^{*}\right\}_{k \in \mathbb{N}} \subset \mathbb{D}^{*}$ such that $z_{k}^{*} \in \mathbb{D}^{*} \backslash$ $\bigcup_{i=1}^{n}\left(D_{1 / k}^{\xi_{i}}\right)^{*}$ for every $k \in \mathbb{N}$. For each $k$, we choose a Möbius transformation $g_{k} \in \operatorname{Möb}(\mathbb{D})$ with $g_{k}(0)=z_{k}$ and define $\mu_{k}=g_{k}^{*} s([\mu])$ and $\nu_{k}=g_{k}^{*} s([\nu])$. Then,

$$
\left\|\left.\left(\mu_{k}-\nu_{k}\right)\right|_{\Delta(0, r)}\right\|_{\infty}=\left\|\left.(s([\mu])-s([\nu]))\right|_{\Delta\left(z_{k}, r\right)}\right\|_{\infty}
$$

tends to 0 as $k \rightarrow \infty$ for any $r>0$. Here, $\Delta(a, r) \subset \mathbb{D}$ denotes a hyperbolic disk with center $a$ and radius $r$.

Since $\left\|\mu_{k}\right\|_{\infty}=\|s([\mu])\|_{\infty}$ and $\left\|\nu_{k}\right\|_{\infty}=\|s([\nu])\|_{\infty}$, by passing to a subsequence, we may assume that $f^{\mu_{k}}$ converges uniformly to some quasiconformal homeomorphism $f^{\mu_{0}} \in \mathrm{QC}(\mathbb{D})$ with a complex dilatation $\mu_{0} \in M(\mathbb{D})$ and that $f^{\nu_{k}}$ converges uniformly to some $f^{\nu_{0}} \in \mathrm{QC}(\mathbb{D})$ with $\nu_{0} \in M(\mathbb{D})$. Let $\lambda_{k}=\mu_{k} * \nu_{k}^{-1}$; that is, $\lambda_{k}$ is the complex dilatation of $f^{\mu_{k}} \circ\left(f^{\nu_{k}}\right)^{-1}$. This satisfies

$$
\left|\lambda_{k} \circ f^{\nu_{k}}\right|=\frac{\left|\mu_{k}-\nu_{k}\right|}{\left|1-\overline{\nu_{k}} \mu_{k}\right|} .
$$

For an arbitrary compact subset $E \subset \mathbb{D}$, we take $r>0$ such that $\left(f^{\nu_{0}}\right)^{-1}(E) \subset$ $\Delta(0, r)$. Since $\left(f^{\nu_{k}}\right)^{-1}$ converges to $\left(f^{\nu_{0}}\right)^{-1}$ uniformly on $\mathbb{D}$ as $k \rightarrow \infty$, we can assume that $\left(f^{\nu_{k}}\right)^{-1}(E) \subset \Delta(0, r)$ for all sufficiently large $k$. Hence,

$$
\left\|\left.\lambda_{k}\right|_{E}\right\|_{\infty} \leqslant \frac{\left\|\left.\left(\mu_{k}-\nu_{k}\right)\right|_{\Delta(0, r)}\right\|_{\infty}}{1-\|\mu\|_{\infty}\|\nu\|_{\infty}} \rightarrow 0 \quad(k \rightarrow \infty) .
$$

Since $E$ is arbitrary, we see from this estimate that the limit $f^{\mu_{0}} \circ\left(f^{\nu_{0}}\right)^{-1}$ of $f^{\mu_{k}} \circ\left(f^{\nu_{k}}\right)^{-1}$ is conformal on $\mathbb{D}$. In fact, $f^{\mu_{0}} \circ\left(f^{\nu_{0}}\right)^{-1}$ is the identity by the normalization. Therefore, $f^{\mu_{0}}=f^{\nu_{0}}$, and both $f^{\mu_{k}}$ and $f^{\nu_{k}}$ converge uniformly to the same limit $f^{\mu_{0}}$ as $k \rightarrow \infty$.

For every $\mu \in M(\mathbb{D})$, we define $\widetilde{\Phi}(\mu)(z)=z^{4} \Phi(\mu)(z)\left(z \in \mathbb{D}^{*}\right)$. As $\rho_{\mathbb{D}^{*}}^{-2}(z)|\Phi(\mu)(z)|$ is bounded, we see that $\widetilde{\Phi}(\mu)$ is a holomorphic function on $\mathbb{D}^{*}$. Similarly to [9, Lemma 6.1], it can be proved that $\widetilde{\Phi}\left(\mu_{k}\right)$ and $\widetilde{\Phi}\left(\nu_{k}\right)$ converge to the same limit $\widetilde{\Phi}\left(\mu_{0}\right)$ locally uniformly on $\mathbb{D}^{*}$ as $k \rightarrow \infty$. Therefore, $\widetilde{\Phi}\left(\mu_{k}\right)-\widetilde{\Phi}\left(\nu_{k}\right)$ converges to 0 , and in particular, $\widetilde{\Phi}\left(\mu_{k}\right)(\infty)-\widetilde{\Phi}\left(\nu_{k}\right)(\infty) \rightarrow 0$ as $k \rightarrow \infty$.

The equivariance of the Bers projection implies that

$$
\Phi\left(\mu_{k}\right)=\Phi\left(g_{k}^{*} \mu\right)=g_{k}^{*} \Phi(\mu) ; \quad \Phi\left(\nu_{k}\right)=\Phi\left(g_{k}^{*} \nu\right)=g_{k}^{*} \Phi(\nu) .
$$

By $\lim _{z \rightarrow \infty} g_{k}(z)=z_{k}^{*}$ and $\lim _{z \rightarrow \infty}\left|z^{2} g_{k}^{\prime}(z)\right|=\rho_{\mathbb{D}^{*}}^{-1}\left(z_{k}^{*}\right)$, it follows that

$$
\begin{aligned}
\rho_{\mathbb{D}^{*}}^{-2}\left(z_{k}^{*}\right)\left|\Phi(\mu)\left(z_{k}^{*}\right)-\Phi(\nu)\left(z_{k}^{*}\right)\right| & =\lim _{z \rightarrow \infty}\left|z^{2} g_{k}^{\prime}(z)\right|^{2}\left|\Phi(\mu)\left(g_{k}(z)\right)-\Phi(\nu)\left(g_{k}(z)\right)\right| \\
& =\left|\widetilde{\Phi}\left(\mu_{k}\right)(\infty)-\widetilde{\Phi}\left(\nu_{k}\right)(\infty)\right| .
\end{aligned}
$$

This tends to 0 as $k \rightarrow \infty$. Since $z_{k}^{*} \in \mathbb{D}^{*} \backslash \bigcup_{i=1}^{n}\left(D_{1 / k}^{\xi_{i}}\right)^{*}$ are arbitrarily chosen, we see that $\Phi(\mu)-\Phi(\nu) \in B_{*}^{X}(\mathbb{D})$.

Here are direct consequences from this theorem.

Corollary 5.2. For every $h \in \mathrm{QS}_{*}^{X}$, the complex dilatation of the barycentric extension $E(h)$ is in $M_{*}^{X}(\mathbb{D})$. Hence, we have a global continuous section $s: T_{*}^{X} \rightarrow$ $M_{*}^{X}(\mathbb{D})$ of the Teichmüller projection $\pi: M_{*}^{X}(\mathbb{D}) \rightarrow T_{*}^{X}$. 
Proof. By setting $\nu=0$ in Theorem [5.1, we obtain that the condition $s([\mu]) \in$ $M_{*}^{X}(\mathbb{D})$ is equivalent to that $\Phi(\mu) \in B_{*}^{X}\left(\mathbb{D}^{*}\right)$. Let $\mu \in M_{*}^{X}(\mathbb{D})$ be the complex dilatation of some quasiconformal extension of $h$. Then, the complex dilatation of the barycentric extension of $h$ is $s([\mu])$. Since $\Phi(\mu) \in B_{*}^{X}\left(\mathbb{D}^{*}\right)$ by Theorem 4.1, we see that $s([\mu]) \in M_{*}^{X}(\mathbb{D})$.

Corollary 5.3. The Teichmüller space $T_{*}^{X}$ is contractible.

Proof. Since $M_{*}^{X}(\mathbb{D})$ is contractible, the assertion follows from Corollary 5.2.

Corollary 5.4. $\beta\left(T_{*}^{X}\right)=\beta(T) \cap B_{*}^{X}\left(\mathbb{D}^{*}\right)$.

Proof. Theorem 4.1 implies that $\beta\left(T_{*}^{X}\right) \subset \beta(T) \cap B_{*}^{X}\left(\mathbb{D}^{*}\right)$. By taking $\nu=0$ in Theorem [5.1, we see that the converse inclusion is also true.

\section{HolOMORPHIC SPLit SUBMERSION}

In this section, we will endow $T_{*}^{X}$ with a complex Banach manifold structure. This is done by investigating the Bers Schwarzian derivative map $\Phi: M_{*}^{X}(\mathbb{D}) \rightarrow$ $B_{*}^{X}\left(\mathbb{D}^{*}\right)$ given in Theorem 4.1 and the section $s$ of $\pi: M_{*}^{X}(\mathbb{D}) \rightarrow T_{*}^{X}$ induced by the barycentric extension in Corollary [5.2. We note that the image of $\Phi$ is $\beta\left(T_{*}^{X}\right)=\beta(T) \cap B_{*}^{X}\left(\mathbb{D}^{*}\right)$ by Corollary 5.4, which is an open subset of $B_{*}^{X}\left(\mathbb{D}^{*}\right)$.

We recall that the right translation $r_{\nu}$ for any $\nu \in M(\mathbb{D})$ defined by $r_{\nu}(\mu)=$ $\mu * \nu^{-1}$ for every $\mu \in M(\mathbb{D})$ is a biholomorphic automorphism of $M(\mathbb{D})$. Concerning the restriction of these automorphisms to $M_{*}^{X}(\mathbb{D})$, we obtain the following result for $r_{\nu}$ given by a trivial Beltrami coefficient $\nu$, which satisfies $\pi \circ r_{\nu}=\pi$ for the Teichmüller projection $\pi: \mu \mapsto[\mu]$.

Lemma 6.1. Let $\nu \in M_{*}^{X}(\mathbb{D})$ such that $[\nu]=[0]$. Then, $r_{\nu}$ is a biholomorphic automorphism of $M_{*}^{X}(\mathbb{D})$.

Proof. We have only to prove that $r_{\nu}(\mu)$ belongs to $M_{*}^{X}(\mathbb{D})$ for every $\mu \in M_{*}^{X}(\mathbb{D})$. The chain rule of complex dilatations implies that

$$
\left|r_{\nu}(\mu) \circ f^{\nu}(z)\right|=\frac{|\mu(z)-\nu(z)|}{|1-\overline{\nu(z)} \mu(z)|}
$$

for $z \in \mathbb{D}$. Then, it suffices to show that the image $f^{\nu}\left(D_{t}^{\xi}\right)$ of a horoball $D_{t}^{\xi}$ for any $\xi \in X$ and $t>0$ is contained in a horoball $D_{t^{\prime \prime}}^{\xi}$ for some $t^{\prime \prime}>0$.

We may consider this problem on the upper half-plane $\mathbb{U}$ under the Cayley transformation $\phi_{\xi}: \mathbb{U} \rightarrow \mathbb{D}$. Then, the horoball $D_{t}^{\xi} \subset \mathbb{D}$ corresponds to $H_{t} \subset \mathbb{U}$. Let $\tilde{f}^{\nu}=\phi_{\xi}^{-1} \circ f^{\nu} \circ \phi_{\xi}$, which extends to the boundary $\mathbb{R}$ as the identity. By some distortion theorem of quasiconformal maps, we can show that there are constants $t^{\prime}, t^{\prime \prime}>0$ depending only on $t>0$ and $\|\nu\|_{\infty}$ with $t^{\prime}, t^{\prime \prime} \rightarrow 0$ as $t \rightarrow 0$ such that $H_{t^{\prime}} \subset \tilde{f}^{\nu}\left(H_{t}\right) \subset H_{t^{\prime \prime}}$, the latter of which is our desired result.

To see this, we take any point $x+i t$ on $\partial H_{t}$ and three other points $x-t, x+t$, and $x-i t$. We may assume that $\tilde{f}^{\nu}$ is a quasiconformal self-homeomorphism of $\mathbb{C}$ by the reflection with respect to $\mathbb{R}$. The cross ratio

$$
\left[z_{1}, z_{2}, z_{3}, z_{4}\right]=\frac{z_{3}-z_{1}}{z_{3}-z_{2}} \cdot \frac{z_{4}-z_{2}}{z_{4}-z_{1}}
$$

for $z_{1}=x-t, z_{2}=x-i t, z_{3}=x+t$, and $z_{4}=x+i t$ equals 2 . If we set $\tilde{f}^{\nu}\left(z_{4}\right)=x^{\prime}+i t^{\prime}$, then $\tilde{f}^{\nu}\left(z_{1}\right)=x-t, \tilde{f}^{\nu}\left(z_{2}\right)=x^{\prime}-i t^{\prime}$, and $\tilde{f}^{\nu}\left(z_{3}\right)=x+t$. By 
simple computation, we have that

$$
\left[\tilde{f}^{\nu}\left(z_{1}\right), \tilde{f}^{\nu}\left(z_{2}\right), \tilde{f}^{\nu}\left(z_{3}\right), \tilde{f}^{\nu}\left(z_{4}\right)\right]=\frac{2 t}{\left(x-x^{\prime}+t\right)+t^{\prime} i} \cdot \frac{2 t^{\prime} i}{\left(x^{\prime}-x+t\right)+t^{\prime} i} .
$$

The distortion theorem of the cross ratio under a quasiconformal map due to Teichmüller (see [2, Chapter III.D] and [14]) implies that the hyperbolic distance between $\left[z_{1}, z_{2}, z_{3}, z_{4}\right]$ and $\left[\tilde{f}^{\nu}\left(z_{1}\right), \tilde{f}^{\nu}\left(z_{2}\right), \tilde{f}^{\nu}\left(z_{3}\right), \tilde{f}^{\nu}\left(z_{4}\right)\right]$ in the 3 -punctured sphere $\mathbb{C}-\{0,1\}$ is bounded by a constant depending only on $\|\nu\|_{\infty}$. If $t^{\prime}$ went to $\infty$ in the above formula with $t$ fixed, then $\left[\tilde{f}^{\nu}\left(z_{1}\right), \tilde{f}^{\nu}\left(z_{2}\right), \tilde{f}^{\nu}\left(z_{3}\right), \tilde{f}^{\nu}\left(z_{4}\right)\right]$ would tend to 0 . This is impossible, and hence by taking the supremum of such $t^{\prime}$ over all $x \in \mathbb{R}$ as $t^{\prime}<\infty$ again, we obtain that $H_{t^{\prime}} \subset \tilde{f}^{\nu}\left(H_{t}\right)$. The other inclusion $\tilde{f}^{\nu}\left(H_{t}\right) \subset H_{t^{\prime \prime}}$ is similarly obtained by considering $\left(\tilde{f}^{\nu}\right)^{-1}$.

We also see that any equivalent Beltrami coefficients $\mu_{1}, \mu_{2} \in M_{*}^{X}(\mathbb{D})$ are mapped to one another by a biholomorphic automorphism $r_{\nu}$ of $M_{*}^{X}(\mathbb{D})$ for some trivial $\nu \in M_{*}^{X}(\mathbb{D})$.

Proposition 6.2. For any $\mu_{1}, \mu_{2} \in M_{*}^{X}(\mathbb{D})$ such that $\left[\mu_{1}\right]=\left[\mu_{2}\right]$, the composition $\nu=\mu_{1}^{-1} * \mu_{2}$ belongs to $M_{*}^{X}(\mathbb{D})$.

Proof. The condition $\nu=\mu_{1}^{-1} * \mu_{2}$ is equivalent to $r_{\nu}\left(\mu_{2}\right)=\mu_{1}$. Then, we have that

$$
\left|\mu_{1} \circ f^{\nu}(z)\right|=\frac{\left|\mu_{2}(z)-\nu(z)\right|}{\left|1-\overline{\nu(z)} \mu_{2}(z)\right|}
$$

for $z \in \mathbb{D}$. Since $[\nu]=0$, the argument in the proof of Lemma 6.1 concerning the image of a horoball by $f^{\nu}$ can also be applied to see that if $\mu_{1}, \mu_{2} \in M_{*}^{X}(\mathbb{D})$, then $\nu \in M_{*}^{X}(\mathbb{D})$.

With the aid of these claims, we can show that the Bers Schwarzian derivative map $\Phi$ is a holomorphic split submersion onto its image. We note that to endow the Teichmüller space with the complex Banach manifold structure, it is enough only to show the existence of a local continuous section to $\Phi$ in our situation (see Corollary 6.4 below).

Theorem 6.3. The Bers Schwarzian derivative $\operatorname{map} \Phi: M_{*}^{X}(\mathbb{D}) \rightarrow B_{*}^{X}\left(\mathbb{D}^{*}\right)$ is a holomorphic split submersion onto its image $\Phi\left(M_{*}^{X}(\mathbb{D})\right)=\beta(T) \cap B_{*}^{X}\left(\mathbb{D}^{*}\right)$.

Proof. Since $\Phi: M(\mathbb{D}) \rightarrow B\left(\mathbb{D}^{*}\right)$ is holomorphic, there is a bounded linear operator $d_{\mu} \Phi: L^{\infty}(\mathbb{D}) \rightarrow B\left(\mathbb{D}^{*}\right)$ at every $\mu \in M(\mathbb{D})$ such that

$$
\frac{\left\|\Phi(\mu+\nu)-\Phi(\mu)-d_{\mu} \Phi(\nu)\right\|_{B}}{\|\nu\|_{\infty}} \rightarrow 0
$$

as $\nu \in L^{\infty}(\mathbb{D})$ tends to 0 . Since $M_{*}^{X}(\mathbb{D})$ and $B_{*}^{X}\left(\mathbb{D}^{*}\right)$ are closed subspaces of $M(\mathbb{D})$ and $B\left(\mathbb{D}^{*}\right)$, respectively, the above convergence at every $\mu \in M_{*}^{X}(\mathbb{D})$ is also valid with restriction of $\Phi$ to $M_{*}^{X}(\mathbb{D})$ and $d_{\mu} \Phi$ to $L_{*}^{X}(\mathbb{D})$. This implies that $\Phi: M_{*}^{X}(\mathbb{D}) \rightarrow B_{*}^{X}\left(\mathbb{D}^{*}\right)$ is holomorphic.

It remains to show that $\Phi$ is a split submersion onto its image $\Phi\left(M_{*}^{X}(\mathbb{D})\right)$. This is equivalent to showing that for every $\mu \in M_{*}^{X}(\mathbb{D})$, there is a holomorphic map $\sigma: U_{\phi} \rightarrow M_{*}^{X}(\mathbb{D})$ defined on some neighborhood $U_{\phi} \subset \Phi\left(M_{*}^{X}(\mathbb{D})\right)$ of $\phi=\Phi(\mu)$ such that $\sigma(\phi)=\mu$ and $\Phi \circ \sigma=\mathrm{id}_{U_{\phi}}$. The existence of some local holomorphic section can be given by a standard argument. This has been carried out in [13] in 
the case where $X$ is a single point set, and we repeat such an argument adapted for our case below.

In order to prove that $\Phi$ is a split submersion, we supplement the proof in 13 . by showing that for any $\mu \in M_{*}^{X}(\mathbb{D})$, a local holomorphic section defined on a neighborhood of $\phi=\Phi(\mu)$ can be chosen so that it sends $\phi$ to $\mu$. We assume that there is a local holomorphic section $\sigma: U_{\phi} \rightarrow M_{*}^{X}(\mathbb{D})$. We set $\nu=\mu^{-1} * \sigma(\phi)$, which belongs to $M_{*}^{X}(\mathbb{D})$ by Proposition 6.2 By Lemma 6.1, $r_{\nu}$ is a biholomorphic automorphism of $M_{*}^{X}(\mathbb{D})$ which satisfies $\pi \circ r_{\nu}=\pi$ and $r_{\nu}(\sigma(\phi))=\mu$. Then, we obtain the required local section $r_{\nu} \circ \sigma$ on $U_{\phi}$.

In the rest of the proof, we show the existence of a local holomorphic section. Let $\phi=\Phi(\mu)$ for a given $\mu \in M_{*}^{X}(\mathbb{D})$. Without loss of generality, we may assume that $\mu=s([\mu])$; that is, $f^{\mu}$ is the barycentric extension of $\left.f^{\mu}\right|_{\mathbb{S}}$. Here, $s: T \rightarrow M(\mathbb{D})$ is the barycentric section which maps $T_{*}^{X}$ into $M_{*}^{X}(\mathbb{D})$ by Corollary [5.2, For the quasiconformal homeomorphism $f_{\phi}=f_{\mu}: \widehat{\mathbb{C}} \rightarrow \widehat{\mathbb{C}}$ that is conformal on $\mathbb{D}^{*}$, we set $D=f_{\phi}(\mathbb{D}), D^{*}=f_{\phi}\left(\mathbb{D}^{*}\right)$, and $\gamma=f_{\phi} \circ j \circ f_{\phi}^{-1}$ for the reflection $j: \zeta \mapsto \zeta^{*}$ with respect to $\mathbb{S}$. We may assume that $f_{\phi}$ is normalized so that $\lim _{z \rightarrow \infty}\left(f_{\phi}(z)-z\right)=0$. Since the barycentric extension $f^{\mu}$ is a bi-Lipschitz diffeomorphism with respect to the hyperbolic metric, we see that so is $\left.f_{\phi}\right|_{\mathbb{D}}$, and hence the quasiconformal reflection $\gamma: D \rightarrow D^{*}$ is a bi-Lipschitz diffeomorphism with respect to the hyperbolic metrics on $D$ and $D^{*}$.

Ahlfors [1] (see also [1, 15]) showed that there exists a constant $C_{1} \geqslant 1$ depending only on $\|\mu\|_{\infty}$ such that

$$
\frac{1}{C_{1}} \leqslant|\gamma(z)-z|^{2} \rho_{D^{*}}^{-2}(\gamma(z))|\bar{\partial} \gamma(z)| \leqslant C_{1}
$$

for every $z \in D$, where $\rho_{D^{*}}(z)$ is the hyperbolic density on $D^{*}$. We set

$$
B_{\varepsilon}(\phi)=\left\{\psi \in B_{*}^{X}\left(\mathbb{D}^{*}\right) \mid\|\psi-\phi\|_{B}<\varepsilon\right\}
$$

for $\varepsilon>0$. For each $\psi \in B_{\varepsilon}(\phi)$, there exists a unique locally univalent holomorphic function $f_{\psi}$ on $\mathbb{D}^{*}$ with the normalization as above such that $\mathcal{S}\left(f_{\psi}\right)=$ $\psi$. Let $g_{\psi}=\left.f_{\psi} \circ f_{\phi}^{-1}\right|_{D^{*}}$. Then, we have that $\mathcal{S}\left(g_{\psi}\right) \circ f_{\phi}\left(f_{\phi}^{\prime}\right)^{2}=\psi-\phi$ and $\sup _{z^{*} \in D^{*}} \rho_{D^{*}}^{-2}\left(z^{*}\right)\left|\mathcal{S}\left(g_{\psi}\right)\left(z^{*}\right)\right|=\|\psi-\phi\|_{B}$.

When $\varepsilon>0$ is sufficiently small, it was proved in [1 that $g_{\psi}$ is univalent (conformal) and can be extended to a quasiconformal homeomorphism of $\widehat{\mathbb{C}}$ whose complex dilatation $\mu_{\psi}$ on $D$ has the form

$$
\mu_{\psi}(z)=\frac{\mathcal{S}\left(g_{\psi}\right)(\gamma(z))(\gamma(z)-z)^{2} \bar{\partial} \gamma(z)}{2+\mathcal{S}\left(g_{\psi}\right)(\gamma(z))(\gamma(z)-z)^{2} \partial \gamma(z)}
$$

Then by (11) and (2), every $\psi \in B_{\varepsilon}(\phi)$ for an even smaller $\varepsilon>0$ satisfies

$$
\left|\mu_{\psi}(z)\right| \leqslant C_{2}\left|\mathcal{S}\left(g_{\psi}\right)(\gamma(z))\right| \rho_{D^{*}}^{-2}(\gamma(z)) \quad(z \in D)
$$

for some constant $C_{2}>0$, which also depends only on $\|\mu\|_{\infty}$. We set $U_{\phi}=B_{\varepsilon}(\phi)$ for this $\varepsilon>0$.

Consequently, $f_{\psi}=g_{\psi} \circ f_{\phi}$ is conformal on $\mathbb{D}^{*}$ and has a quasiconformal extension to $\widehat{\mathbb{C}}$ whose complex dilatation $\nu_{\psi}$ on $\mathbb{D}$ is given as

$$
\nu_{\psi}=\frac{\mu+\left(\mu_{\psi} \circ f_{\phi}\right) \tau}{1+\bar{\mu}\left(\mu_{\psi} \circ f_{\phi}\right) \tau}, \quad \tau=\frac{\overline{\partial f_{\phi}}}{\partial f_{\phi}} .
$$


It is well known that $\nu_{\psi}$ depends holomorphically on $\psi$. Now it follows from (3) that

$$
\begin{aligned}
\left|\mu_{\psi}\left(f_{\phi}(\zeta)\right)\right| & \leqslant C_{2}\left|\mathcal{S}\left(g_{\psi}\right)\left(\gamma\left(f_{\phi}(\zeta)\right)\right)\right| \rho_{D^{*}}^{-2}\left(\gamma\left(f_{\phi}(\zeta)\right)\right) \\
& =C_{2}\left|\mathcal{S}\left(g_{\psi}\right)\left(f_{\phi}(j(\zeta))\right)\right| \rho_{D^{*}}^{-2}\left(f_{\phi}(j(\zeta))\right) \\
& =C_{2}|\psi(j(\zeta))-\phi(j(\zeta))| \rho_{\mathbb{D}^{*}}^{-2}(j(\zeta)) \\
& =C_{2}\left|\psi\left(\zeta^{*}\right)-\phi\left(\zeta^{*}\right)\right| \rho_{\mathbb{D}^{*}}^{-2}\left(\zeta^{*}\right)
\end{aligned}
$$

for every $\zeta \in \mathbb{D}$ with $\zeta^{*}=j(\zeta) \in \mathbb{D}^{*}$.

Since $\psi, \phi \in B_{*}^{X}\left(\mathbb{D}^{*}\right)$, the above estimate implies that $\mu_{\psi} \circ f \in M_{*}^{X}(\mathbb{D})$. Then, we see from (4) that $\nu_{\psi} \in M_{*}^{X}(\mathbb{D})$. Since $\Phi\left(\nu_{\psi}\right)=\psi$, we conclude that $\sigma: U_{\phi} \rightarrow$ $M_{*}^{X}(\mathbb{D})$ defined by $\sigma(\psi)=\nu_{\psi}$ is a local holomorphic section to $\Phi$. This completes the proof.

Corollary 6.4. The Bers embedding $\beta: T_{*}^{X} \rightarrow B_{*}^{X}\left(\mathbb{D}^{*}\right)$ is a homeomorphism onto the domain $\beta(T) \cap B_{*}^{X}\left(\mathbb{D}^{*}\right)$ in $B_{*}^{X}\left(\mathbb{D}^{*}\right)$. Hence, the Teichmüller space $T_{*}^{X}$ has the complex structure modeled on the complex Banach space $B_{*}^{X}\left(\mathbb{D}^{*}\right)$. Under this complex structure, the projection $\pi: M_{*}^{X}(\mathbb{D}) \rightarrow T_{*}^{X}$ is also a holomorphic split submersion.

Proof. By the continuity of $\Phi: M_{*}^{X}(\mathbb{D}) \rightarrow B_{*}^{X}\left(\mathbb{D}^{*}\right)$, we see that $\beta$ is continuous. In the other direction, the existence of the local continuous section to $\Phi$ shown in Theorem 6.3 together with the continuity of the projection $\pi$ ensures the continuity of the inverse $\beta^{-1}: \beta(T) \cap B_{*}^{X}\left(\mathbb{D}^{*}\right) \rightarrow T_{*}^{X}$. These facts prove that $\beta$ is a homeomorphism onto the image.

Finally, we note that the corresponding result to Proposition 3.1 is also valid for the space of the holomorphic quadratic differentials.

Proposition 6.5. For any $X=\left\{\xi_{1}, \ldots, \xi_{n}\right\} \subset \mathbb{S}, B_{*}^{X}\left(\mathbb{D}^{*}\right)=B_{*}^{\xi_{1}}\left(\mathbb{D}^{*}\right)+\cdots+$ $B_{*}^{\xi_{n}}\left(\mathbb{D}^{*}\right)$.

Proof. For the Bers Schwarzian derivative map $\Phi: M_{*}^{X}(\mathbb{D}) \rightarrow B_{*}^{X}\left(\mathbb{D}^{*}\right)$, we consider its derivative $d_{0} \Phi: L_{*}^{X}(\mathbb{D}) \rightarrow B_{*}^{X}\left(\mathbb{D}^{*}\right)$ at $0 \in M_{*}^{X}(\mathbb{D})$. By Proposition 3.1, $L_{*}^{X}(\mathbb{D})=$ $L_{*}^{\xi_{1}}(\mathbb{D})+\cdots+L_{*}^{\xi_{n}}(\mathbb{D})$. Since $d_{0} \Phi$ is a linear map, we see that

$$
\begin{aligned}
d_{0} \Phi\left(L_{*}^{X}(\mathbb{D})\right) & =d_{0} \Phi\left(L_{*}^{\xi_{1}}(\mathbb{D})+\cdots+L_{*}^{\xi_{n}}(\mathbb{D})\right) \\
& =d_{0} \Phi\left(L_{*}^{\xi_{1}}(\mathbb{D})\right)+\cdots+d_{0} \Phi\left(L_{*}^{\xi_{n}}(\mathbb{D})\right) \\
& =B_{*}^{\xi_{1}}\left(\mathbb{D}^{*}\right)+\cdots+B_{*}^{\xi_{n}}\left(\mathbb{D}^{*}\right) .
\end{aligned}
$$

Since $\Phi$ is a submersion by Theorem 6.3, $d_{0} \Phi: L_{*}^{X}(\mathbb{D}) \rightarrow B_{*}^{X}\left(\mathbb{D}^{*}\right)$ is surjective, namely, $d_{0} \Phi\left(L_{*}^{X}\left(\mathbb{D}^{*}\right)\right)=B_{*}^{X}\left(\mathbb{D}^{*}\right)$. This completes the proof of Proposition 6.5.

\section{ACKNowledgment}

The authors would like to thank the referee for a careful reading of the manuscript and for giving valuable suggestions. 


\section{REFERENCES}

[1] Lars V. Ahlfors, Quasiconformal reflections, Acta Math. 109 (1963), 291-301, DOI 10.1007/BF02391816. MR154978

[2] Lars V. Ahlfors, Lectures on quasiconformal mappings, manuscript prepared with the assistance of Clifford J. Earle, Jr., Van Nostrand Mathematical Studies, No. 10, D. Van Nostrand Co., Inc., Toronto, Ont.-New York-London, 1966. MR.0200442

[3] Kari Astala and Michel Zinsmeister, Teichmüller spaces and BMOA, Math. Ann. 289 (1991), no. 4, 613-625, DOI 10.1007/BF01446592. MR:1103039

[4] A. Beurling and L. Ahlfors, The boundary correspondence under quasiconformal mappings, Acta Math. 96 (1956), 125-142, DOI 10.1007/BF02392360. MR86869

[5] Lennart Carleson, On mappings, conformal at the boundary, J. Analyse Math. 19 (1967), 1-13, DOI 10.1007/BF02788706. MR215986

[6] Guizhen Cui, Integrably asymptotic affine homeomorphisms of the circle and Teichmüller spaces, Sci. China Ser. A 43 (2000), no. 3, 267-279, DOI 10.1007/BF02897849. MR1766456

[7] Adrien Douady and Clifford J. Earle, Conformally natural extension of homeomorphisms of the circle, Acta Math. 157 (1986), no. 1-2, 23-48, DOI 10.1007/BF02392590. MR857678

[8] Clifford J. Earle, Frederick P. Gardiner, and Nikola Lakic, Asymptotic Teichmüller space. II. The metric structure, In the tradition of Ahlfors and Bers, III, Contemp. Math., vol. 355, Amer. Math. Soc., Providence, RI, 2004, pp. 187-219, DOI 10.1090/conm/355/06452. MR2145063

[9] Clifford J. Earle, Vladimir Markovic, and Dragomir Saric, Barycentric extension and the Bers embedding for asymptotic Teichmüller space, Complex manifolds and hyperbolic geometry (Guanajuato, 2001), Contemp. Math., vol. 311, Amer. Math. Soc., Providence, RI, 2002, pp. 87-105, DOI 10.1090/conm/311/05448. MR.1940165

[10] Richard Fehlmann, Über extremale quasikonforme Abbildungen (German), Comment. Math. Helv. 56 (1981), no. 4, 558-580, DOI 10.1007/BF02566227. MR656212

[11] Frederick P. Gardiner and Nikola Lakic, Quasiconformal Teichmüller theory, Mathematical Surveys and Monographs, vol. 76, American Mathematical Society, Providence, RI, 2000. MR.1730906

[12] Frederick P. Gardiner and Dennis P. Sullivan, Symmetric structures on a closed curve, Amer. J. Math. 114 (1992), no. 4, 683-736, DOI 10.2307/2374795. MR1175689

[13] Hu Yun, Wu Li, and Shen Yuliang, On symmetric homeomorphisms on the real line, Proc. Amer. Math. Soc. 146 (2018), no. 10, 4255-4263, DOI 10.1090/proc/14018. MR 3834655

[14] Irwin Kra, On Teichmüller's theorem on the quasi-invariance of cross ratios, Israel J. Math. 30 (1978), no. 1-2, 152-158, DOI 10.1007/BF02760836. MR.508260

[15] Olli Lehto, Univalent functions and Teichmüller spaces, Graduate Texts in Mathematics, vol. 109, Springer-Verlag, New York, 1987. MR867407

[16] Katsuhiko Matsuzaki, The universal Teichmüller space and diffeomorphisms of the circle with Hölder continuous derivatives, Handbook of group actions. Vol. I, Adv. Lect. Math. (ALM), vol. 31, Int. Press, Somerville, MA, 2015, pp. 333-372. MR.3380337

[17] Subhashis Nag, The complex analytic theory of Teichmüller spaces, Canadian Mathematical Society Series of Monographs and Advanced Texts, John Wiley \& Sons, Inc., New York, 1988. MR.927291

[18] Stephen W. Semmes, Quasiconformal mappings and chord-arc curves, Trans. Amer. Math. Soc. 306 (1988), no. 1, 233-263, DOI 10.2307/2000836. MR927689

[19] Yuliang Shen and Huaying Wei, Universal Teichmüller space and BMO, Adv. Math. 234 (2013), 129-148, DOI 10.1016/j.aim.2012.10.017. MR3003927

[20] Leon A. Takhtajan and Lee-Peng Teo, Weil-Petersson metric on the universal Teichmüller space, Mem. Amer. Math. Soc. 183 (2006), no. 861, viii+119, DOI 10.1090/memo/0861. MR 2251887

[21] H. Wei and K. Matsuzaki, Symmetric and strongly symmetric homeomorphisms on the real line with non-symmetric inversion, arXiv:1905.05933 2019. 
Department of Mathematics and Statistics, Jiangsu Normal University, Xuzhou 221116, People's Republic of China

Email address: hywei@jsnu.edu.cn

Department of Mathematics, School of Education, Waseda University, Shinjuku, TOKYO 169-8050, JAPAN

Email address: matsuzak@waseda.jp 